\title{
Déconstruire le « carno-phallogocentrisme » : l'écoféminisme comme critique de la rationalité occidentale
}

\author{
Janne Burgart Goutal
}

Très souvent, on réduit l'écologie à une lutte contre les dégradations environnementales dues aux excès du capitalisme technologique, et le féminisme à un combat contre la persistance d'inégalités entre les sexes. Cette réduction rend incompréhensible l'écoféminisme : qu'ont à voir de tels objectifs? N'est-il pas artificiel, voire incohérent de les relier? Mais une telle critique se méprend sur le type d'écologie et de féminisme à l'oeuvre dans l'écoféminisme. Dans la préface de Gaia and God, Rosemary Radford Ruether définit l'écoféminisme comme la conjonction du féminisme radical et de l'écologie profonde : il s'agit d'aller au-delà de l'analyse des effets (surexploitation des ressources, pollution, extinction massive d'espèces, réchauffement climatique d'un côté; inégalités économiques, politiques, sociales entre les sexes, de l'autre), et d'en rechercher les racines profondes. À ce niveau d'analyse, on découvrirait des connexions souterraines entre domination sur les femmes et la nature (et aussi dominations de race et de classe), qui convergeraient en une seule et même «matrice de domination », un unique «système » ancré dans un unique «paradigme» sous-jacent. C'est une telle thèse que nous souhaitons explorer ici : quel peut bien être ce système? Comment penser les connexions entre les différentes formes de domination, sans pour autant les confondre grossièrement en une unité indistincte?

C'est essentiellement autour du concept de «carnophallogocentrisme », forgé par Derrida en 1989, que tournera notre lecture de l'écoféminisme. Afin d'écarter de possibles malentendus, justifions d'emblée ce choix qui ne va pas de soi. Pourquoi privilégier ce terme, alors qu'il n'y a pas (à notre connaissance) d'écrit écoféministe conséquent qui le reprenne à son compte, les textes écoféministes parlant plutôt de «logique de domination » (Warren), de «culture de la mise à distance » (Starhawk), de «phallocratisme » (d'Eaubonne) ou de «patriarcat capitaliste » (Mies et Shiva)? Parce que sans nier la pertinence politique des concepts internes au corpus écoféministe, le terme de «carno-phallogocentrisme » semble particulièrement approprié pour nommer de façon synthétique, et sous un angle explicitement 
philosophique, la logique de domination que l'écoféminisme met au jour, qu'il déconstruit et qu'il cherche à dépasser. Loin de marginaliser la contribution de l'écoféminisme, nous souhaitons donc, en nous concentrant sur ce concept, souligner la portée philosophique majeure de ce mouvement : comme nous tenterons de le montrer, attaquer le « carnophallogocentrisme », c'est peut-être attaquer le schème paradigmatique de la rationalité occidentale en son cœur même. Derrida écrit en effet : «je voudrais un jour démontrer que ce schème implique la virilité-carnivore. Je parlerais d'un carno-phallogocentrisme » (Derrida, «Manger» 294). Ce programme philosophique crucial, dont il ne cesse de dire qu'il faudrait « un jour » s'y atteler, c'est l'écoféminisme qui l'accomplit dans les faits - même si, curieusement, Derrida n'évoque jamais d'auteure de cette mouvance ni de dette à son égard... ( $c f$. Fraiman)

Nous l'examinerons ici à partir de quatre questions :

1. Comment s'est progressivement construit et affiné le diagnostic écoféministe sur le «système» liant toutes les formes de domination, jusqu'à aboutir à une conception synthétisable sous le terme de « carno-phallogocentrisme »?

2. Quels sont le sens et la pertinence de ce concept? Pourquoi et comment relier les trois notions de logocentrisme, phallocentrisme et «carnivorisme » qui, à priori, n'ont pas grand chose à voir les unes avec les autres?

3. À travers ce concept, que vise-t-on: un travers particulier à la civilisation occidentale ou une structure commune à toute civilisation, voire inhérente à la conscience humaine?

4. Enfin, en quoi un tel concept éclaire-t-il non seulement les rapports de domination explicitement visés (sur les femmes, les peuples et classes exploités, les animaux, la nature...), mais plus largement la tradition philosophique occidentale? Pourquoi peut-on affirmer que le carno-phallogocentrisme est l'«architecture secrète» de la pensée occidentale, le fondement caché d'une «logique imperturbable, à la fois grecque et abrahamique (judéo-christianoislamique) [dont] nous ne cessons de vérifier l'invariance jusque dans notre Modernité » (Derrida, Animal 40)?

L'enjeu de ce questionnement sera d'évaluer l'ambition constamment affichée par l'écoféminisme de constituer un "nouveau paradigme », susceptible de dépasser enfin le fond misogyne et écocide de la civilisation occidentale. Peut-on vraiment lire l'écoféminisme non pas comme un courant particulier au sein de la pensée occidentale, mais comme son renversement? 


\section{Du « patriarcat » au « carno-phallogocentrisme »}

Dès sa naissance, l'écoféminisme se démarque du féminisme et de l'écologie mainstream en reliant les questions du sexisme et de la crise environnementale. Son pari est d'aller au-delà d'analyses locales et isolées, et de construire un cadre explicatif systémique. Pour lui,

les séparations confortables ne sont plus opérantes. [...] Car même si on nous a appris que les problèmes sont distincts, que le viol est distinct de la guerre nucléaire, que la lutte d'une femme pour l'égalité de salaire n'est reliée ni à celle de l'adolescent noir pour trouver un travail ni à celle contre l'exportation d'un réacteur nucléaire vers un site failles sismiques près de volcans en activité aux Philippines, toutes ces réalités sont formées par la même conscience qui modèle nos relations de pouvoir. (Starhawk 29)

Une constante de l'écoféminisme est donc la recherche de « connexions » entre les oppressions qui selon lui font système : il s'agit en particulier d' ' explorer comment la domination des femmes et la domination de la nature sont interconnectées, à la fois dans les idéologies culturelles et les structures sociales » (Ruether, Gaia and God 2); mais pas exclusivement: c'est "l'imbrication du sexisme avec toutes les autres formes d'oppression », nommément le racisme, le classisme, l'antisémitisme, le colonialisme et le spécisme, qui l'intéresse. D'emblée, l'interpénétration des dominations est posée : c'est ce nœud qui fait l'objet de l'écoféminisme.

En un sens, il s'agit de l'élargissement d'une intuition que l'on trouve déjà chez Beauvoir. Celle-ci souligne dans la préface au Deuxième sexe l'analogie qui existe entre toutes les formes de discriminations sociales : "Qu'il s'agisse d'une race, d'une caste, d'un sexe réduits à une condition inférieure, les processus sont les mêmes. L'"éternel féminin" c'est l'homologue de 1'“âme noire" et du "caractère juif". » (Beauvoir t. I, 27) L'écoféminisme étend cette idée de relation entre les dominations en montrant que les espèces et réalités non-humaines sont elles aussi prises dans le même schéma : "Se fondant sur l'intuition du féminisme socialiste selon laquelle le racisme, le classisme et le sexisme sont interconnectés, les écoféministes ont découvert des relations entre ces formes d'oppression humaine et les structures oppressives du spécisme et de l'antinaturalisme. » (Gaard, «Queer Ecofeminism »137)

Mais quel est ce système qui unit dans un même destin les femmes, les «races » et peuples colonisés, les classes sociales exploitées et «la nature » — c'est-à-dire les animaux, les plantes, les écosystèmes, l'environnement et aussi tout ce qui est défini comme nature en nous : corps, besoins, pulsions, émotions? Au fil de son évolution, le diagnostic de l'écoféminisme à ce sujet se précise, dessinant une étiologie de plus en plus fine. 
Sous l'influence du féminisme radical et culturel des années 1970, la plupart des premiers écrits écoféministes se contentent d'une analyse monofocale, incriminant purement et simplement le patriarcat : Mary Daly fustige ainsi le «pouvoir patriarcal », « androcrate » ou «masculiniste», « les principautés du Patriarcat » et leur «luxure phallique » destructrice de toute vie, chargée de « haine envers la femme, envers la nature » (Daly 9); Françoise d'Eaubonne rend le «système mâle » ou «phallocratisme » responsable de la domination croisée de la nature, des femmes et autres catégories infériorisées - la «civilisation technologique superurbaine et superindustrielle » n'en étant que le dernier avatar (d'Eaubonne, Féminisme $228 s q$.).

Mais rapidement, l'insuffisance d'une telle explication unilatérale devient patente. Ainsi, dès 1975, Ruether refuse toute «analyse monolithique » incapable de rendre raison de l'intersection des dominations, qui fait justement l'objet de l'écoféminisme. Comme le souligne Val Plumwood, «la réduction par la critique féministe de la source du mal à une identité simplement mâle tend à obscurcir les vrais problèmes politiques; nous devons donc remplacer le concept de phallo(go)centrisme par un concept plus complexe» (Plumwood, Feminism 68).

C'est surtout à partir de la fin des années 1980 et dans les années 1990 que de tels concepts apparaissent. Maria Mies et Vandana Shiva incriminent le «capitalisme patriarcal », articulant ainsi sexe, classe et race, et complexifiant l'analyse féministe par un angle matérialiste d'inspiration marxiste, renouvelé par les études postcoloniales et l'altermondialisme. Quant à Starhawk, Karen Warren et Plumwood, elles font du patriarcat un symptôme d'un problème plus profond, ancré dans un « cadre conceptuel oppressif » qu'elles appellent respectivement « mise à distance » (Starhawk), « logique de domination » (Warren) ou «modèle de maître »(Plumwood). Derrière ces nouveaux concepts se trouve le même refus de l'exclusivité, et même de la primauté, d'un seul axe critique. L'enjeu est de dépasser l'explication monocausale, dans un souci inclusif de combinaison des différentes «plaques tectoniques de la théorie de la libération »(Plumwood, Feminism 3). Il s'agit de ne pas commettre une erreur symétrique à celle de Marx : en analysant la domination sociale sous le seul angle de la lutte des classes, il s'est rendu aveugle à la « lutte des sexes » sans laquelle, pourtant, le capitalisme est incompréhensible ( $c f$. Federici). L'écoféminisme, s'il veut vraiment constituer un modèle d'explication global de la domination en tant que système, sans pour autant gommer la multiplicité des rapports de domination, doit se mettre en quête d'une articulation non réductionniste de leurs causalités et des théories s'y rapportant.

Derrière cette identification adéquate du nœud qui lierait ensemble et génèrerait tous les systèmes d'oppression, se profile une analyse d'une 
ampleur philosophique plus vaste. C'est Carolyn Merchant qui franchit le pas décisif : d'après Greta Gaard, The Death of Nature (1980) est le premier ouvrage à ne pas se contenter d'étudier les liens entre formes d'oppressions (racisme, sexisme, classisme, spécisme, antisémitisme, antinaturalisme), mais à formuler leur ancrage dans la rationalité scientifique et économique moderne en général: «Le point le plus provocant de son ouvrage est d'affirmer une relation intersectionnelle entre le racisme, le spécisme, le colonialisme, le capitalisme et le modèle mécaniste de la science moderne. » (Gaard, «Ecofeminism Revisited » 28) Dès lors, l'écoféminisme va pouvoir occuper une place de choix dans le grand projet de déconstruction de la rationalité moderne — voire de la rationalité occidentale depuis ses origines — qui anime la philosophie contemporaine au moins depuis Nietzsche et qui irrigue des courants majeurs comme la théorie critique de l'école de Francfort, la philosophie postmoderne ou encore les études postcoloniales et décoloniales. Ce ne serait pas à cause d'errements ponctuels et contingents, de dérives historiques malencontreuses, que la civilisation occidentale aurait développé des tendances inégalitaires, dominatrices, sexistes et écocides; au contraire, de telles tendances en seraient intrinsèquement constitutives. Dès Descartes, voire dès Platon, aurait été scellé un «mariage de la raison avec la domination » (Plumwood, Feminism 72), qui constituerait le cœur caché de tous les systèmes philosophiques, socioéconomiques et idéologiques dominants de l'Occident.

C'est là que l'écoféminisme rejoint Derrida, arrivé à la même analyse par un autre chemin. Il soutient en effet que la «question de l'animal » et celle de la différence (ou plutôt « différance ») des sexes sont 1 '« indispensable fil conducteur pour lire les philosophes et accéder à une sorte d'architecture secrète de la construction, donc de la déconstruction d'un dispositif discursif, d'une cohérence, sinon d'un système » caractéristique de notre civilisation (Derrida, Animal 147). Cette « logique imperturbable à la fois grecque et abrahamique (judéo-christianoislamique) » (40), qui grèverait l'ensemble de la pensée occidentale depuis ses sources platoniciennes et bibliques, et que Derrida débusque minutieusement dans les écrits de Descartes, Hobbes, Kant, Heidegger, Lacan et Levinas (entre autres), Derrida commence par la nommer « logocentrisme ». Mais dans L'animal que donc je suis (1997), il «note très vite en passant, au titre de l'autobiographie intellectuelle, que le déconstruction du "logocentrisme" a dû, tout nécessairement, se déployer avec les années en déconstruction du "phallogocentrisme", puis du "carnophallogocentrisme" » (144).

Ainsi, que l'on parte d'une critique du «phallocratisme » comme la créatrice du mot «écoféminisme » (d'Eaubonne), ou bien d'une critique du logocentrisme comme celle de Derrida, il semble que l'on soit conduit nécessairement à dévoiler une logique sous-jacente à la pensée occidentale 
dominante, une «configuration à la fois systématique et hiérarchique » (Derrida, Bête 55) reliant en un tout la souveraineté du logos, la suprématie oppressante et dominatrice du phallus et le «carnivorisme » (que l'on peut en première approche définir comme haine ou négation de la nature et de l'animalité). Le nouage inextricable de ces trois tendances constituerait «le socle» camouflé de la rationalité occidentale, telle qu'elle s'exprime dans nos grands textes philosophiques, religieux, juridiques, scientifiques, dans notre histoire et dans l'organisation de nos sociétés.

\section{Carno- / phallo- / logo- : quels rapports?}

Mais la pertinence et la portée d'un tel concept ne vont pas de soi : que signifie-t-il? Parler de « carno-phallogocentrisme » revient à affirmer qu'il existe un lien nécessaire - et non pas une coïncidence contingente

- entre logocentrisme, phallocentrisme et «carnivorisme». Mais pourquoi y aurait-il une connexion (voire une équivalence) entre ces trois tendances qui n'ont, à première vue, aucun rapport entre elles? N'est-il pas artificiel de postuler leur relation intrinsèque?

Commençons par décomposer ce terme afin de le clarifier. On peut tout d'abord définir le logocentrisme comme le «mariage de la raison et de la domination » (Plumwood), consacré par Platon et célébré depuis par (quasiment) toute la tradition philosophique occidentale : il consiste à ériger le logos, «la raison », en faculté maîtresse de l'esprit humain et en attribut fondant toute supériorité, légitimant la domination sur les êtres et entités définis comme non rationnels. Ce concept de logocentrisme revient à révéler la dimension offensive, voire politique, du rationalisme.

Le phallocentrisme peut être défini comme l'identification (implicite) du sujet au mâle humain, qui a pour corrélat l'objectivation et l'infériorisation de tous les autres êtres; il se manifeste dans la dimension patriarcale de nos sociétés, dans la misogynie ou «gynophobie » (Lederer) de la pensée occidentale, et plus subtilement dans son androcentrisme sciences humaines et naturelles confondues (comme le montrent, par exemple, Judith Butler au sujet de la psychanalyse et de l'anthropologie ou Donna Haraway au sujet de la biologie et de la primatologie).

Enfin, le «carnivorisme » prend ici un sens symbolique, et non seulement littéral : en employant ce terme, Derrida veut surtout «parler ici du "manger" métonymique » (Derrida, « Manger» 297). Il désigne par là la construction normative de l'identité humaine (du concept dominant de l'humain, c'est-à-dire, au fond, du concept de l'humain comme dominant) par l'ingestion plus ou moins ritualisée d'animaux non humains, dont on se démarque ainsi, et par le sacrifice de tout ce qui en nous est associé à l'animalité : notre corps, nos besoins et pulsions, notre sensibilité... deux mécanismes qui seraient liés. Nous vivons dans des «cultures [...] 
pour lesquelles le sacrifice carnivore est essentiel, comme l'être-chair » (293). En effet, comme le montre également Carol Adams, ce « carnivorisme » à la fois réel et symbolique définit dans notre culture le «schème qui domine le concept de sujet. Celui-ci ne se veut pas seulement maitre et possesseur actif de la nature; dans nos cultures, il accepte le sacrifice et mange de la chair» (295). Interprété symboliquement, cela signifie que le sujet occidental conquiert son statut d'humain "véritable », "civilisé », au prix du renoncement, de la négation, voire de la destruction métaphorique et réelle de la nature en lui et hors de lui : notre anthropologie, notre conception de la nature, notre rapport à l'animalité et aux animaux sont fondés sur une "structure sacrificielle». C'est ainsi, par exemple, que Georges Bataille affirme, d'une façon typique de ce « carnivorisme » dit « humaniste»:

Je pose en principe un fait peu contestable : que l'homme est l'animal qui n'accepte pas simplement le donné naturel, qui le nie. [...] L'homme parallèlement se nie lui-même, il s'éduque, il refuse par exemple de donner à la satisfaction de ses besoins animaux ce cours libre, auquel l'animal n'apporte pas de réserve. Il est nécessaire encore d'accorder que les deux négations que, d'une part, l'homme fait du monde donné et, d'autre part, de sa propre animalité, sont liées. (Bataille 288 sq.)

Or, selon les analyses d'Adams et de Derrida, on pourrait aller jusqu'à qualifier toute «la tradition judéo-christiano-islamique de guerre contre l'animal, une guerre sacrificielle aussi vieille que la Genèse » (Derrida, Animal 140). Elle se déclinerait sous de multiples formes au cours de l'histoire : dans l'Antiquité, cette guerre «carnivore » prend le visage de la «somatophobie» (Spelman) ou «haine de la vie» (Nietzsche) tristement fameuse du platonisme, ou encore de l'« érotophobie » (Gaard) biblique. Dans la modernité, elle se manifeste par exemple dans « l'indifférence mécaniste » (Derrida, Animal 140) de Descartes réduisant la nature à un royaume, dont nous devons nous rendre « comme maitres et possesseurs », et les animaux à des machines inertes et insensibles; elle se prolonge dans la «haine et la détestation vouée par le kantien à l'animalité », qui définit avec une «perversité profonde » la morale et l'humanisation comme «sacrifice de notre animalité » (141); ou encore dans le spécisme et «l'impératif différentiel » (Plumwood) qui traversent avec une constance obstinée la pensée occidentale, ses philosophies, ses sciences sociales et naturelles...

On peut faire remonter à Nietzsche la critique conjointe du logocentrisme et du «carnivorisme » (sous la forme de la «haine de la vie », en l'occurrence) comme traits typiques de la civilisation occidentale; la difficulté est d'y articuler la notion de phallocentrisme. Car le sens de cette notion de «carno-phallogocentrisme» est bien l'idée d'une intrication de ces trois modes de domination «centriste », nœud qui constituerait le socle occulté de la rationalité occidentale. 
Mais il ne suffit pas d'affirmer vaguement qu' «il existe des connexions » entre rationalisme dominateur (logocentrisme), dévaluation oppressive des femmes (phallocentrisme) et dégradation, voire haine sacrificielle de la nature, de l'animalité et des animaux («carnivorisme ») : encore faut-il expliquer précisément quels sont ces liens. Dans Ecofeminism and Globalization, Heather Eaton et Lois Ann Lorentzen les résument autour de trois grands axes :

1. Premièrement, il existerait des liens empiriques: comme le montrent les études sur la justice environnementale, les femmes, catégorie sociale défavorisée, souvent exclues de la propriété privée et vouées aux tâches de subsistance (un aspect du phallocentrisme), sont les premières victimes de la dégradation de l'environnement naturel (un effet concret du «carnivorisme») engendrée par les assauts de la rationalité technoscientifique et économique (manifestation moderne du logocentrisme): «Le prix de la destruction de ressources est externalisé et divisé de manière inégale entre les divers groupes économiques de la société, et ces coûts sont supportés en grande partie par les femmes. » (Mies et Shiva 91) Ce cercle vicieux entre la détérioration de l'environnement et celle de la condition féminine au nom d'une certaine «rationalité » technoscientifique et économique apparaîtrait avec une netteté particulière lors des phases d'essor du capitalisme: $\mathrm{au} \mathrm{XVII}^{\mathrm{e}}$ siècle en Europe (la chasse aux sorcières symbolisant ce lien, aux yeux de nombreuses écoféministes) et aujourd'hui partout où il s'exporte sous l'effet des politiques internationales de «maldéveloppement » (Shiva).

2. Deuxièmement, il existerait des liens épistémologiques, qui se manifesteraient clairement au cœur de la science moderne. En effet, conformément à l'épistémologie féministe du «point de vue » (Hartsock, Harding) ou des «savoirs situés» (Haraway), les écoféministes mettent en évidence l'importance de l'expérience vécue dans la construction de tout discours, même ceux qui se qualifient de scientifiques. Or, une des caractéristiques des groupes dominants, en particulier masculins, auxquels appartiennent les intellectuels et scientifiques des sociétés modernes, est qu'ils se préservent des tâches reproductives et de subsistance, les confiant à des subordonnés, alors que ce sont de telles tâches qui mettent en contact de façon vivante et sensible avec la nature en nous (soins du corps) et hors de nous (recherche, production, transformation des ressources nécessaires à la vie). Ils seraient dès lors condamnés à adopter une perspective abstraite et désincarnée, privée de tout rapport concret avec la réalité matérielle, en particulier naturelle. C'est pourquoi les écoféministes affirment que les grands partispris conceptuels, axiologiques et méthodologiques de la physique et 
de la biologie modernes, désignés comme responsables de la «mort de la nature » (Merchant), sont liés à des biais de genre et à une construction patriarcale de la science. Réduction de la nature, des animaux et des corps à une matière inerte, à une ressource manipulable, rationalisme instrumental étroit et désincarné, mécanisme abstrait, conception et pratique du savoir comme rapport unilatéral entre un sujet actif et un objet passif, exclusion de la sensibilité morale au nom de l'«objectivité » - bref, « rupture et dissection de ce qui constitue un tout vivant» (Mies et Shiva 29): tous ces postulats centraux d'une science sans conscience reflèteraient la «masculinité abstraite » (Chodorow) de savants que les rapports sociaux de genre rendent étrangers, donc aveugles et indifférents aux symbioses et dynamiques du monde des corps et de la vie. Cela vaut également pour les sciences sociales : une illusion androcentriste les empêcherait de saisir le rôle des femmes dans les processus environnementaux, inspirant ainsi des théories et politiques doublement pernicieuses (socialement et écologiquement).

3. Enfin, il existerait aussi des liens conceptuels : en effet, comme le déplorait déjà Beauvoir, dans les dualismes qui structurent la pensée occidentale, les hommes sont associés à la culture (esprit, raison, transcendance) et les femmes à la nature (corps, émotions, immanence), laquelle est également conçue comme opposée à la raison. Par conséquent, la dévaluation des femmes et celle de la nature vont de pair, rejaillissant l'une sur l'autre en un cercle vicieux : elles s'articulent avec la survalorisation de la raison, posée comme leur contraire et maîtresse. Le logocentrisme est donc au cœur de la dévaluation des femmes (qui seraient émotives et irrationnelles) et des animaux (tenus pour privés de raison); c'est une clé de voûte de la «connexion entre phallocentrisme et anthropocentrisme »(Plumwood, Feminism 10). Ces articulations conceptuelles sont analysées avec un grand soin par Plumwood: «Le rapport des dualismes avec la perspective du maître apparaît dans beaucoup de sources anciennes. [...] Par exemple, Aristote, dans un célèbre passage des Politiques justifiant l'esclavage, met en relation ces dualismes afin de justifier la domination de l'humain sur la nature, de l'homme sur la femme, du maître sur l'esclave et de la raison sur le corps et les émotions » (46), au titre que les seconds de chaque paire seraient dépourvus de raison.

À force de mettre en évidence ces liens habituellement cachés entre logocentrisme, phallocentrisme et «carnivorisme», les écrits écoféministes en viennent parfois à donner l'impression que ces trois tendances n'en sont en fait qu'une seule. Derrida lui-même dit que le terme de carno-phallogocentrisme, loin d'être un composé hétéroclite, est 
une «tautologie », une «synthèse a priori » (Derrida, «Manger » 294). Mais peut-on vraiment identifier ses trois composantes? Comment concevoir la forme logique de leur relation?

Les premiers écrits écoféministes se contentent souvent d'affirmer leur identité; cela revient à dire que «c'est une même matrice de domination sociale » (Ruether, New Woman XVII) qui unit en un destin commun (infériorisation, subordination, exploitation, agression, voire destruction) les femmes et les êtres naturels (ainsi que les peuples et classes dominés). Comme nous l'avons vu, cette identité est posée sur un mode réductionniste chez d'Eaubonne et Daly (« le patriarcat»). Derrida aussi affirme une identité pure et simple entre les trois tendances nouées : ce ne seraient que trois noms pour un même mécanisme vu sous différents angles, trois synonymes.

Cependant, il nous semble impossible de les amalgamer ainsi; il y a là une simplification du problème, confusément rabattu sur une entité indistincte («la domination», «le mal / mâle»). Or, il faut nécessairement les distinguer, car il serait faux d'affirmer que toutes les civilisations patriarcales sont écocides ou encore que toutes les civilisations respectueuses de la nature le sont des femmes. C'est d'ailleurs une critique récurrente contre les formes simplistes d'écoféminisme : leur idéalisation des sociétés vivant « en harmonie avec la nature » occulte souvent l'oppression des femmes qui peut y régner (comme le montrent Eaton et Lorentzen au sujet des indigènes du Chiapas ou du Japon traditionnel). Logocentrisme, phallocentrisme et «carnivorisme» sont donc trois tendances distinctes, mais articulées. Comment le sont-elles? Au fil de l'évolution de l'écoféminisme, les manières de penser cette articulation deviennent plus précises.

Un premier concept employé pour préciser les distinctions entre les différents axes de domination est celui d'analogie. Une analogie est une équivalence entre des rapports : en l'occurrence, il s'agit d'affirmer que le rapport homme / femme et le rapport humain / nature (ou culture / nature, ou raison/ nature), sont similaires, structurellement équivalents. D'Eaubonne écrit ainsi : «le rapport de l'homme à la nature est plus que jamais celui de l'homme à la femme » (d'Eaubonne, Écologie/Féminisme 15); et Sherry Ortner insinue que, pour la pensée occidentale, «la femme est à l'homme ce que la nature est la culture » (Ortner 68). Ce qui rend ces rapports analogues, c'est essentiellement qu'ils sont oppressifs, « coloniaux » et dualistes : dans tous ces cas, «l'une des deux parties est toujours considérée comme supérieure, se développant et progressant toujours aux dépens de l'autre » (Mies et Shiva 17). Mais à son tour, une telle manière de penser le lien entre les différents axes de dominations est insatisfaisante. L'idée d'analogie est trop floue : dire que deux rapports se ressemblent ne suffit pas à montrer qu'ils sont vraiment reliés, qu'ils dépendent l'un de l'autre. 
C'est pourquoi, à partir des années 1980, les écoféministes vont essayer de dégager des relations de causalité entre domination des femmes et de la nature. La question se pose alors de savoir laquelle de ces deux formes de domination est la cause de l'autre. De ce point de vue, deux grandes réponses se dégagent: les écoféministes inspirées par l'écologie sociale de Murray Bookchin (Ruether, Ynestra King, Chaia Heller) reprennent à ce dernier l'idée selon laquelle «les divisions entre la société et la nature trouvent leurs racines les plus profondes à l'intérieur du domaine social, dans les conflits installés de longue date qui opposent les humains les uns aux autres » (Bookchin 47). Interprétant cette idée sous un angle féministe, Ruether affirme ainsi que « le concept de domination de la nature a été forgé, à l'origine, sur la domination sociale exercée par des groupes maîtres sur des groupes esclaves, dont la plus fondamentale est la relation entre hommes et femmes » (Ruether, New Woman 204). Ce serait donc la relation hommes/ femmes, déjà marquée du sceau du dualisme, de l'injustice et de l'oppression (phallocentrisme), qui aurait modelé la relation à la nature et qui serait donc la cause profonde de la domination sur la nature et les animaux (« carnivorisme »). Un des intérêts de cette thèse est de révéler que notre rapport à la nature est politique. C'est parfois également sur une argumentation inspirée de la psychanalyse que s'appuie cette conception causale : ainsi Ruether, associant dans un esprit jungien concepts psychanalytiques et analyses mythologiques, explique-t-elle que c'est de la crainte jalouse des hommes vis-à-vis de la toute-puissance primordiale de la Mère, source de vie, qu'est issu le patriarcat. Celui-ci a assimilé la puissance génératrice des femmes à un chaos à contrôler, ce qui aurait dès lors contaminé notre rapport à la nature fertile. Ariel Salleh renchérit à partir d'une "phénoménologie genrée » (Salleh 38) : "si les hommes ne peuvent "créer" la vie, ils peuvent se 1'“approprier" » (39). Ce serait de ce «nœud psycho-sexuel explosif» (54), ancré dans la différence des sexes, que naîtrait le rapport masculiniste à la nature, fondé sur l'appropriation, la conquête et le désir de maîtrise. Les jeux de mots «M/Other » (Salleh) et «Mat $(\mathrm{t})$ er » (Mies, Subsistence) résument cette thèse: c'est du rapport complexe des humains, et en particulier des hommes, à la mère, que dériverait leur rapport délétère à la nature.

Inversement, pour les auteures inspirées par l'existentialisme beauvoirien, c'est l'infériorisation première de la nature qui est cause de l'oppression des femmes, par le biais de l'assimilation de ces dernières à une nature déjà dévaluée. Beauvoir écrit en effet : «L'humanité a toujours cherché à s'évader de sa destinée spécifique [...]. C'est parce que l'humanité se met en question dans son être, c'est-à-dire préfère à la vie des raisons de vivre, qu'en face de la femme l'homme s'est posé comme le maître » (Beauvoir 117). Ce serait donc parce que «la société humaine est une anti-phusis » (98) qu'elle est aussi anti-femmes; ce serait de la volonté de se distinguer de la nature, de l'animalité, du règne de la matière et de 
l'en-soi, afin de conquérir le statut de sujet, de pour-soi authentiquement humain (bref, du «carnivorisme » et du logocentrisme inhérents à la conscience humaine), que découleraient le phallocentrisme et le patriarcat. Dans une certaine mesure, Starhawk reprend cette explication, en affirmant que le problème «vient en fait de la question de la relation au corps. [...] En tant qu'être humains, notre relation au corps est toujours ambivalente. Le corps a des limites inhérentes. Les traits propres au corps féminin $[\ldots]$ ont été utilisés pour nous rejeter, nous exploiter, nous dévaloriser. » (Starhawk $11 s q$.)

Qu'il ait pour origine la dévaluation des femmes ou celle de la nature, du point de vue empirique, ce lien causal s'exprime de façon circulaire: il $y$ a un cercle vicieux entre la détérioration de l'environnement ou le rejet du corps (deux formes d'antinaturalisme) et la dégradation de la condition féminine. C'est justement pour rendre raison de cette causalité circulaire et pour résoudre le débat sans fin qui oppose la thèse de la primauté du rapport hommes / femmes à celle de la primauté du rapport humanité / nature, que les écrits écoféministes des années 1990 vont proposer une nouvelle manière de penser la relation entre ces deux structures de domination. Il ne s'agirait ni d'identité uniforme, ni de vague analogie, ni de causalité linéaire, mais bien d'un rhizome, d'un « réseau », d'une «structure emboîtée », d'un «système fait de multiples nœuds » (Plumwood, Feminism 42). Plumwood développe tout particulièrement ce modèle à partir d'une critique du réductionnisme impliqué par l'explication causale unilatérale. Elle reproche ainsi à Bookchin et à ses disciples de soutenir que «la domination de la nature découle de la domination d'humains par des humains et en est entièrement dérivée; ce faisant, ils affirment une thèse historique réductionniste » (14). Selon elle, une telle explication causale est certes meilleure que les analyses totalement apolitiques de l'écologie profonde de Warwick Fox, mais elle reste insuffisante, au même titre que toute explication monocausale. La théorie dérivée de l'existentialisme beauvoirien, qui fonde la domination des femmes sur la dévaluation de la nature, prête donc le flanc à la même critique. Or, on ne peut donner la primauté à l'un des schèmes oppressifs liés dans le carno-phallogocentrisme, car ils se constituent réciproquement, d'emblée et inextricablement: du fait du «caractère genré du dualisme nature/culture » (4), la naturalisation des femmes (et autres dominés) et la féminisation de la nature (et autres dominés) s'imbriquent originairement; et l'on ne peut pas séparer ni hiérarchiser les arrière-plans antinaturaliste et gynophobe des concepts dominants de raison et de sujet, qui s'entre-constituent. Autrement dit, ce n'est pas telle ou telle forme de domination qui est première, mais leur nœud même. C'est donc «tout un réseau complexe de dualismes interconnectés » (4) qu'il faut déconstruire. 
Dans ce modèle du réseau élaboré par Plumwood, les dualismes interdépendants s'articulent au moyen de « postulats charnières ». Il s'agit d'un ensemble de métaphores, de glissements sémantiques, d'analogies et d'associations d'idées, qui en nouent les termes respectifs. Ils évoluent selon les auteurs et les époques, se cumulant en strates qui s'entrerenforcent au fil du temps. Il ne s'agit donc pas d'une «matrice de domination » établie une fois pour toutes, dont les termes seraient fixés depuis la nuit des temps ni même plus modestement depuis les écrits fondateurs de la civilisation occidentale; au contraire, Plumwood a la finesse d'en retracer l'évolution au cours des siècles. Elle montre ainsi comment Platon, Descartes, Rousseau, Kant, Hegel et Marx font résonner, chacun à leur manière propre, des variations autour du «dualisme genré raison / nature ». Sa thèse est que ce réseau de dualismes interdépendants se développe en plusieurs stades

formés par le pouvoir et correspondant à différentes étapes du processus d'accumulation: ce processus a tout d'abord donné naissance aux dualismes sacré/ profane (puisque le pouvoir était essentiellement religieux), mâle / femelle et maître / esclave. Des stades postérieurs du processus d'accumulation ont occasionné de nouvelles formes de dualismes, généralement nées de nuances, d'inflexions nouvelles des formes plus anciennes. Ainsi, la période coloniale a produit le dualisme civilisé / primitif à titre de déclinaison des dualismes raison / nature, raison / animal et âme / corps; et l'essor de la science moderne y a lié le dualisme sujet / objet. (44)

\section{Un paradigme universel?}

Ce réseau de dualismes oppressifs formant le carnophallogocentrisme, l'écoféminisme ne se contente pas de le mettre au jour: il cherche à le dépasser. Sa «question devient: comment renversons-nous non pas ceux qui sont actuellement au pouvoir, mais le principe du pouvoir-sur? Comment donnons-nous forme à une société fondée sur le principe du pouvoir-du-dedans? [...] Par un changement de paradigme, de conscience. » (Starhawk 28 et 34) Mais on peut se demander s'il n'y a pas une forme de naïveté dans ce projet. Plus profond et insidieux qu'un simple travers occidental particulier et contingent, le carno-phallogocentrisme ne serait-il pas une structure inhérente à la conscience humaine comme telle, un invariant anthropologique inévitable et indépassable?

Une telle question nous confronte à un dilemme délicat. D'un côté, il semble illusoire d'imaginer que ce système de domination soit exclusivement occidental et que les autres civilisations échappent à cette structuration misogyne, anthropocentriste et antinaturaliste. Toute société ne classe-t-elle pas le réel en le dichotomisant et en posant des hiérarchies entre les êtres, surtout selon des critères de sexe et d'espèce? Mais d'un 
autre côté, n'y aurait-il pas une forme d'impérialisme ethnocentriste à postuler l'universalité de notre mode de penser et de dualiser le réel?

L'idée selon laquelle le carno-phallogocentrisme, c'est-à-dire la domination croisée des femmes et de la nature au nom de la raison, est un fait universel, traverse de nombreux écrits, aussi bien au sein qu'au dehors de l'écoféminisme ou du féminisme. Ainsi, lorsque Beauvoir se demande : «D'où vient que ce monde a toujours appartenu aux hommes? » (Beauvoir 24), elle répond: «ce n'est pas en donnant la vie, c'est en risquant sa vie que l'homme s'élève au-dessus de l'animal; c'est pourquoi dans l'humanité la supériorité est accordée non au sexe qui engendre mais à celui qui tue. Nous tenons ici la clef de tout le mystère. »(115) Autrement dit, le carno-phallogocentrisme serait ancré au plus profond de la conscience humaine, dans les formes à priori de la subjectivité : c'est en niant la nature, l'animalité en lui et hors de lui (« carnivorisme ») et par conséquent la féminité qui y est associée (phallocentrisme), au profit d'une identification avec la raison et la transcendance (logocentrisme), que l'être humain se constituerait en authentique sujet. Beauvoir appuie cette thèse sur une relecture de la dialectique hégélienne du maître et de l'esclave : le malheur de la femme, "c'est d'avoir été biologiquement vouée à répéter la Vie, alors qu'à ses yeux mêmes la Vie ne porte pas en soi ses raisons d'être, et que ces raisons sont plus importantes que la vie même.» (116) Elle invoque également des sources historiques et ethnologiques supposées attester l'universalité de ce paradigme: «La catégorie de l'Autre est aussi originelle que la conscience elle-même. Dans les sociétés les plus primitives, dans les mythologies les plus antiques on trouve toujours une dualité qui est celle du Même et de l'Autre [...]; c'est ce qui ressort entre autres des travaux de Granet sur la pensée chinoise, de ceux de Dumézil sur les Indes et Rome. » (18) L'anthropologie structurale naissante lui fournit également des arguments. Lévi-Strauss en effet affirme dans Les structures élémentaires de la parenté que la culture humaine en tant que telle se constitue contre la nature, et que les femmes, du fait de la puissance d'engendrement de leur corps, sont universellement associées à la nature. Puisque selon lui toute civilisation tend de toutes ses forces à se démarquer de la nature (impératif différentiel qui est au cœur $\mathrm{du}$ « carnivorisme»), cela fait que le pouvoir social et politique est, par définition, masculin: c'est contre la puissance naturelle des femmes qu'émergerait le pouvoir culturel, qui reviendrait donc par définition aux hommes. Il y aurait donc, entre les trois composantes du carnophallogocentrisme, un lien essentiel, nécessaire et universel. Le projet de le dépasser serait donc illusoire : autant espérer que l'humanité ne soit plus ce qu'elle est, et que la civilisation même, en tant que telle, renie ce qui la fonde.

Cependant, les avancées plus récentes de l'ethnologie remettent en question cette thèse en l'attaquant sur plusieurs fronts. En effet, d'une part, 
on peut douter de l'universalité du «carnivorisme », c'est-à-dire à la fois de la dévaluation de la nature - qui semble au contraire valorisée et même sacralisée par de nombreux peuples —, et du rôle essentiel du sacrifice des animaux et de l'animalité dans la construction symbolique normative de l'identité humaine — improbable dans les peuples qui conçoivent humains et non-humains comme formant un grand système social d'alliances et de parentés (car même s'ils chassent, ils ne nient pas notre affinité avec les animaux ni notre animalité, donc). On peut également contester l'affirmation selon laquelle la conscience humaine associerait universellement les femmes et la nature (liant donc universellement phallocentrisme et «carnivorisme») - bref, l'universalité de ce que Salleh appelle «l'équation subliminale Homme/Femme = Nature » (Salleh 13), le «complexe $\mathrm{H} / \mathrm{F}=\mathrm{N} »$ ou « régime $\mathrm{H} / \mathrm{F}=\mathrm{N}$ » (57). Ainsi, Françoise Héritier, bien qu'adhérant à l'idée d'universalité de la "valence différentielle des sexes », qu'elle tient pour un pilier structurel de toute société (au même titre que la prohibition de l'inceste et l'exogamie), indique pourtant, au passage, qu'on ne peut universaliser l'identification des femmes à la nature et des hommes à la culture, postulée par Beauvoir et Lévi-Strauss ou encore Daly et Griffin. Elle évoque en effet le peuple Inuit, chez qui «le froid, le cru, la nature sont du côté de l'homme, le chaud, le cuit, la culture, du côté de la femme » (Héritier 222). De même, Lorentzen et Eaton expliquent que chez les Indiens du Chiapas, les hommes sont considérés comme plus proches de la nature du fait de leurs activités agricoles qui les mettent en contact direct avec la terre, tandis que les femmes sont davantage classées du côté de la culture car elles sont chargées des activités artisanales, de la transformation des produits naturels et de la transmission des coutumes et du langage (Eaton et Lorentzen 61). Plus radicalement encore, les travaux de Nicole-Claude Mathieu remettent en cause l'idée d'universalité de la domination masculine en montrant qu'il s'agit moins d'une réalité que d'une interprétation récurrente de l'ethnologie occidentale, qui découle en fait, paradoxalement, d'un point de vue androcentriste, incapable de s'intéresser aux sphères d'activité féminines, de saisir leur importance et le pouvoir qui en émane. Une telle cécité trahirait en outre un biais ethnocentriste : ce seraient les habitudes de pensée inculquées par nos sociétés patriarcales qui expliqueraient que nous projetons un tel postulat sur les autres sociétés.

Il semble donc qu'on doive abandonner l'idée selon laquelle le carno-phallogocentrisme, ennemi fondamental de l'écoféminisme, serait une tare universelle, inhérente à «la » conscience humaine et à toute civilisation. Plumwood écrit ainsi : «le dualisme a souvent été considéré comme un aspect inévitable de la condition humaine, en particulier dans la tradition existentialiste, qui présente sa structure comme une dimension maudite mais irrémédiable de la subjectivité. [...] Je conteste l'affirmation de son inévitabilité. »(Plumwood, Feminism 61) Voilà qui ouvre un 
espoir de changement pour l'écoféminisme... tout en redéfinissant la portée de sa critique.

$\mathrm{Si}$, comme nous venons de le montrer, la dévaluation et l'oppression conjointes des femmes, des animaux non humains et de la nature au nom de la raison ne sont pas universelles, il est toutefois tentant de se demander, de façon plus fine, s'il n'existerait pas une corrélation invariante entre les trois paramètres articulés dans le concept de carnophallogocentrisme, considérés de façon «neutre », c'est-à-dire abstraction faite de toute notion de domination; à savoir : un certain type de rapport hommes / femmes, un certain mode de rapport humains / nature et une certaine forme de logos. On pourrait ainsi faire l'hypothèse d'une interdépendance entre (a) la façon dont une société pense et organise la différence des sexes (patriarcat ou matriarcat, sociétés matrilinéaires ou patrilinéaires, matrilocales, uxorilocales ou patrilocales...), (b) la façon dont elle se rapporte à la nature, structure ses relations avec son environnement, s'oriente au sein de la diversité des étants humains et non humains et enfin (c) son type de logos ou de «rationalité » (logique propositionnelle indo-européenne, catégories de la «pensée sauvage » étudiées par Lévi-Strauss, logiques inclusives extrême-orientales...). Y at-il une correspondance, dans chaque société, entre la construction de la différence des sexes, le rapport à l'environnement naturel et la forme de logique en vigueur? Dans une optique écoféministe, l'intérêt de cette question serait de déterminer si, par exemple, certaines logiques favorisent la domination sur les femmes, les animaux et la nature ou au contraire induisent des relations sociales et environnementales respectueuses; ou encore, si une certaine organisation des rapports entre les sexes rejaillit sur les modes de penser, de classer et de se rapporter à l'environnement naturel et aux autres espèces. Cela offrirait des clefs pour engager la «mutation »systémique que ce mouvement appelle ardemment.

En couplant seulement deux de ces trois paramètres, de nombreux auteurs en viennent effectivement à affirmer une corrélation. Ainsi, les tenants de l'écologie profonde affirment souvent un lien entre la logique régulant la pensée d'une civilisation et son rapport à la nature plus ou moins respectueux ou destructeur. C'est pourquoi, comme le souligne John Baird Callicott, les écologistes ont souvent puisé aux logiques orientales non dualistes et non substantialistes : Emerson et Thoreau se sont inspirés non seulement de l'éthique mais aussi de la logique hindouiste; les écologistes des années 1960 (Suzuki, Snyder) se sont tournés vers les modes de pensée paradoxaux du zen; ceux des années 1980 (Muir), vers les formes logiques mouvantes du taoïsme; Naess se réfère constamment au bouddhisme (Callicott 35)... Une telle démarche repose sur l'idée qu'il existe bien un lien entre les règles du logos par lesquelles on conduit la raison et la façon dont on se rapporte à la nature : telle logique engendrerait telle cosmologie; telle manière de penser et 
classer de façon oppositionnelle ou au contraire inclusive conditionnerait tels rapports entre humains et non-humains. Descola évoque lui aussi un lien entre la logique occidentale, qui fonctionne selon le modèle de la «classification par attributs», par «dichotomies successives de traits contrastifs » opposés (Descola 332), et notre cosmologie dualiste (qu'il appelle «naturaliste ») : ce ne serait pas un hasard si une logique reposant sur l' «opposition contrastive », le principe de contradiction et le tiersexclu coexiste en Occident avec une cosmologie violemment scindée, posant un «Grand Partage » entre humains et non-humains, culture et nature. Inversement, il indique que les cultures qui ne séparent pas nature et culture, mais qui vivent leur unité sur un mode soit «animiste » (les Amérindiens) soit «totémique » (les Aborigènes d'Australie), développant à l'égard des non-humains des relations de parenté respectueuse, ont aussi une autre logique classificatoire : elles procèdent par «catégorisation prototypique », établissant des «taxinomies inclusives fondées sur la généralisation prototypique d'une saillance perceptive », où le « processus d'identification » (332) ne repose pas sur une logique oppositionnelle.

Parallèlement, certains auteurs affirment une corrélation entre deux autres des trois paramètres en jeu, à savoir le type de logique en vigueur dans une culture et l'organisation sociale qui y prévaut, en particulier en matière de rapports des sexes. En effet, la différence des sexes serait un point clé, voire l'origine même des notions d'identité et de différence (qui sont les fondements de la logique); car « la distinction entre les sexes est, presque par définition, la première de toutes les distinctions » (Douglas 154), donc peut-être leur matrice, ainsi que celle de tous les modes de classification :

La différence sexuée [...], j'y vois la base objective et irrécusable d'un système englobant de classification selon l'identique et le différent. [...] Cette catégorisation de base est à mes yeux issue de l'observation liminaire de la différence sexuée sur laquelle la volonté humaine n'a pas de prise. Elle est au cœur de tous les systèmes de pensée de toutes les sociétés. [...] La différence anatomique visible entre ce qui est mâle et ce qui est femelle est le butoir permettant à l'esprit d'appréhender des différences indubitables et irréfutables sous la forme d'une opposition princeps entre identique et différent sur laquelle vont se construire toutes les autres. (Héritier 15 sq. et 128)

Ce serait alors d'une certaine manière de penser l'identité et la différence entre les sexes (en insistant soit sur leur continuité, soit sur leur opposition, soit sur leur asymétrie, soit sur leur complémentarité, etc.) que découleraient les règles de logique d'une culture. Autrement dit, même la logique serait secrètement politique - plus précisément, genrée. Derrida appuie cette hypothèse, au sujet de la logique occidentale en l'occurrence, en rappelant l'étymologie commune des mots «même » et «maître » ou « époux » dans les langues indo-européennes : il est frappant que le «mot signifiant "maître" (potis; en sanskrit patih: maître et époux; en grec 
posis : époux; en composition despotês) en vienne à [...] signifier "luimême" » (Derrida, Bête vol. I, 101); cela montrerait que la «valeur d'ipséité, l'ipse », bref la position du même et de l'autre, de l'identique et du différent, est intrinsèquement liée dans notre logique à des rapports de pouvoir, en particulier sexués, «comme si le pouvoir était d'abord reconnu à celui qu'on pouvait désigner ou qui pouvait se désigner le premier comme le même »(101). Une telle analyse permet de conforter l'hypothèse d'une correspondance entre le type de logique et les rapports sociaux de sexes en vigueur dans une société, au moins concernant notre civilisation. Il faudrait donc examiner précisément si d'autres formes de logique - celles, « inclusives », «non-propositionnelles », « nondualistes» ou encore dites «sauvages», qui structurent différentes langues et philosophies extra-occidentales — sont corrélées à d'autres manières de penser et de pratiquer les rapports sociaux de sexes. Nous ne sommes pas en mesure de développer ce point ici, mais on pourrait déjà noter que la logique non dualiste et non substantialiste qui structure les langues et modes de pensée chinois et japonais, refusant les principes de contradiction et de tiers-exclu, va de pair avec une conception du masculin et du féminin comme imbriqués et complémentaires, comme l'exprime le symbole du Yin et du Yang - conception qui s'avère bien plus égalitaire, voire favorable au féminin (Yin). Ainsi le Tao-Te-King exhorte : «Connais en toi le masculin; adhère au féminin »; et le shintoïsme pose la supériorité de la Nigi-mi-tama, partie féminine de l'esprit, sur l'Ara-mi-tama, partie masculine de l'esprit ( $c f$. Eaton et Lorentzen).

Il serait cependant hasardeux de prétendre qu'une telle conception symbolique, fondée sur une logique non dualiste, entraîne une valorisation concrète des femmes : la Chine et le Japon n'en sont pas moins des sociétés patriarcales. À fortiori, l'articulation des trois paramètres (logique, cosmologie ou rapport à la nature et rapports des sexes) pose de difficiles problèmes. En effet, si comme le montre Descola, les concepts même de nature et de culture sont des constructions propres à la modernité occidentale, on ne peut pas analyser en ces termes les peuples du passé ou d'ailleurs: leurs catégories, leurs associations d'idées, leurs liens conceptuels, leurs manières d'associer et de dissocier, de classer les identités et les différences (notamment entre hommes et femmes, humains et non-humains, raison et déraison) ne sont pas superposables aux nôtres. Douglas met d'ailleurs en garde contre la tentation d'établir systématiquement des corrélations entre ces paramètres : certes, «dans diverses cultures, les théories en vigueur sur les forces cosmiques confèrent un rôle plus ou moins explicite à l'énergie sexuelle. [...] [Mais] il serait vain de vouloir établir la relation entre les grandes lignes de ces variations métaphysiques, d'une part, et les différences d'organisation sociale, d'autre part. » (159) Par conséquent, on peut douter que les trois paramètres reliés dans le concept de carno-phallogocentrisme, même 
dégagés de la notion de domination, aient des connexions nécessaires et universelles.

Que reste-t-il alors du concept de carno-phallogocentrisme? Toutes ces objections et relativisations, induites notamment par l'ethnologie, doivent-elles conduire à rejeter comme infondée l'affirmation centrale de l'écoféminisme, selon laquelle il existe un étroit réseau de liens - et pas seulement une coïncidence contingente et insignifiante - entre phallocentrisme, logocentrisme et « carnivorisme »?

\section{Une clé de déconstruction de la rationalité occidentale}

En réalité, malgré les nuances et précisions qu'il nous a fallu introduire par souci de rigueur, le concept de carno-phallogocentrisme n'en reste pas moins, à nos yeux, un outil original de déconstruction critique de la civilisation et de la rationalité occidentales. C'est ainsi que l'utilise, par exemple, Plumwood, évitant par là de nombreux écueils évoqués ci-dessus, lorsqu'elle affirme : «Le caractère genré du dualisme nature / culture et de tout le réseau de dualismes qui y sont interconnectés n'est pas un trait de la pensée humaine ou de la culture en soi, [...] mais une caractéristique spécifique à la pensée occidentale » (Plumwood, Feminism 4) ou, plus précisément encore, à la «forme dominante [master form] de la rationalité occidentale » (I). S'il ne peut servir de fondement à une théorie totalisante de «la domination » (et c'est tant mieux), le concept de carno-phallogocentrisme pourrait donc contribuer à cette entreprise majeure de la philosophie contemporaine, permettant ainsi à l'écoféminisme d'occuper une place de choix aux côtés de la théorie critique, de la philosophie postmoderne et des études postcoloniales et décoloniales. Loin de ne recouvrir qu'une préoccupation théorique, il y a là un enjeu profondément politique, car «analyser sans fin et dans ses intérêts toute la machinerie conceptuelle» de notre culture (Derrida, «Manger» 288) est indispensable pour créer une «philosophie de l'avenir » ( $c f$. Nietzsche), inventer des « cadres conceptuels » libérateurs, forger « un discours restructuré pour tenter de situer autrement la question de ce qu'est, peut être, doit être un sujet humain, une morale, un droit, une politique du sujet humain » (Derrida, «Manger» 289). Alors, en quoi le concept de carno-phallogocentrisme permet-il de dévoiler les ressorts et rouages secrets de la «machinerie conceptuelle» occidentale, afin de tracer des chemins de traverse pour la dépasser et initier une «mutation » systémique de nos modes de pensée, des rapports entre les sexes, les peuples, les espèces et avec la nature?

Répondre à cette question suppose de définir précisément ce que les écoféministes entendent par «rationalité occidentale»: quel sens donner à cette expression sans nier la diversité des philosophies et des théories au cours de l'histoire de l'Occident — bref, sans tomber dans 
l'essentialisme? On peut sans simplification excessive, nous semble-t-il, dégager trois grands traits de la forme de rationalité typique de l'Occident, relativement invariants ou tout du moins nettement dominants au fil du temps, au-delà des immenses divergences théoriques entre auteurs, disciplines et courants; ils imprègnent, en filigrane, tous les domaines de pensée, ce sont donc eux qui traceraient cette «logique imperturbable» sous-jacente que vise l'écoféminisme. Il s'agit d'une part d'une métaphysique substantialiste, ou «de la présence » (contrairement, par exemple, à la métaphysique taoïste du flux et du devenir); d'autre part, d'une logique fondée sur les principes d'identité, de non-contradiction et de tiers-exclu, qui conduit à penser le même et l'autre sur un mode dualiste, en opposition disjonctive (contrairement, par exemple, à la logique taoïste de la non-dualité ou à la «logique pertinente»); et, enfin, d'une anthropologie ou philosophie du sujet, articulée autour de l'idée de raison. Mais en quoi cette métaphysique, cette logique et cette anthropologie persistantes fonderaient-elles une civilisation oppressive, notamment pour les femmes, les animaux et la nature? La question se pose, car selon l'écoféminisme, derrière leur apparence inoffensive, c'est bien sur elles que reposeraient en dernière instance les dominations et les rapports de colonisation dénoncés. Cela vaut même pour l'écoféminisme matérialiste: Mies, par exemple, dénonce non seulement des injustices empiriques, mais aussi les «biais conceptuels » qui les induisent ou qui du moins les consolident et les pérennisent en les ancrant profondément dans nos systèmes mentaux et sociaux (cf. Patriarchy). En effet, comme nous l'avons indiqué en introduction, une des spécificités de ce courant est qu'il entend aller au-delà des simples effets que sont pour lui la crise environnementale, l'exploitation des animaux, la colonisation, la discrimination des femmes et d'autres "minorités ", pour remonter aux racines du mal. Or, si ces racines plongent au plus profond de notre civilisation, c'est qu'elles doivent nécessairement s'ancrer dans ces invariants culturels obstinés - ou même, plus exactement, dans quelque chose qui constituerait leur fond commun. Mais de quoi s'agit-il?

Un certain nombre d'écoféministes (dont Starhawk et Plumwood) avancent le diagnostic suivant: le dualisme. Fondamentalement, c'est cette déformation caricaturale et oppressive de la différence qui rejaillirait de façon pernicieuse dans nos anthropologies, nos métaphysiques, nos religions et aussi nos sociétés, nos économies et nos politiques: «L'histoire de la civilisation patriarcale pourrait être lue comme un effort cumulatif pour briser ce lien, pour séparer l'esprit et la chair, la nature et la culture, l'homme et la femme. [...] Cette rupture fonde les oppressions inséparables de race, de sexe, de classe, et la destruction écologique.» (Starhawk 19) Entrons plus précisément dans l'analyse de cette «séparation» dualiste. On peut partir du constat qu'une donnée fondamentale de la réalité consiste dans la diversité : biodiversité des espèces, diversité culturelle des peuples, diversité sociale des individus, 
groupes et sexes... Cet infini pullulement de différences (cette irréductible «différance »), la conscience éprouve le besoin de l'ordonner; toute culture élabore ainsi une certaine logique, avec ses catégories, ses formes de classification et ses règles logiques. Comme nous l'avons vu en évoquant notamment Descola, ces logiques peuvent organiser la diversité de façon oppositionnelle ou non, hiérarchisée ou non, en forgeant des dichotomies exclusives ou en insistant sur les « saillances prototypiques » communes et les relations inclusives, etc. Or, le choix de la pensée occidentale a été de privilégier une logique dualiste - une «logique du 1/0 », comme l'appelle Salleh (Ecofeminism 35). «Davantage qu'une simple dichotomie, différence ou non-identité, davantage aussi qu'une simple relation hiérarchique »(Plumwood, Feminism 47), le dualisme consiste à caricaturer la différence en opposition exclusive et hiérarchisée; il est donc au cœur du «cadre conceptuel oppressif» dénoncé par l'écoféminisme. C'est par excellence le paradigme par lequel la logique occidentale, en croyant ou en prétendant penser la différence et la diversité, les nie et les trahit en fait. Hegel, déjà, mettait en évidence sous le nom de «logique d'entendement» l'incapacité de la logique occidentale, dès Parménide, à faire droit à la différence, immédiatement ramenée à l'identité ou déformée en opposition disjonctive, du fait des principes d'identité, de non-contradiction et de tiers-exclu qui la structurent : «L'être est et il n'est pas possible qu'il ne soit pas [...]; quant au non-être, il n'est rien », écrit ainsi Parménide de façon caractéristique (Parménide 94). Comme le montre Plumwood, le dualisme revient à dégrader la diversité, au plan empirique comme conceptuel, au moyen de cinq mécanismes déformants qu'elle décortique minutieusement: l'«hyperséparation» des termes comparés (par exemple, hommes et femmes, humains et animaux, culture et nature, esprit et corps...); la mise à l'arrière-plan de celui des termes considéré comme inférieur; son « incorporation » (définition relationnelle, par subordination); son instrumentalisation; et son homogénéisation. Du fait de cette déformation de la diversité en opposition exclusive, hiérarchique, homogénéisante, «la structure logique du dualisme constitue le fondement principal de la connexion entre les formes d'oppression »(Plumwood, Feminism 3). À la fois cadre mental et social, elle biaise à notre insu notre rapport au monde, ancrant profondément dans nos consciences et nos inconscients une vision scindée du réel, une négation insidieuse de toute forme d'égalité dans la diversité.

Le dualisme constitue ainsi la matrice logique du carnophallogocentrisme, qui consiste bien à déformer la différence des sexes, la diversité des espèces et la multiplicité des facultés mentales en oppositions disjonctives, asymétriques et oppressives, placées respectivement sous l'égide infondée du masculin, de l'humain et de la raison. Le carnophallogocentrisme ne constitue donc pas une anomalie isolée et insignifiante : il reflète et exprime le fond logique général de la rationalité 
occidentale dans son ensemble. Car le dualisme qui le sous-tend constitue aussi la logique génératrice des grands postulats typiques, ou du moins absolument dominants de la pensée occidentale, tels que nous les avons dégagés plus haut : ainsi la métaphysique de la substance pose un primat de l'identité à soi (l'«être ») sur la différence, du Même sur l'Autre, conformément à la logique dualiste; la philosophie du sujet «humaniste » construit sa définition de l'homme sur une logique d'opposition dualiste entre humanité et animalité, raison et nature, homme et femme. On saisit donc que c'est un seul et même paradigme logique qui sous-tend le carnophallogocentrisme et les fondements métaphysiques et anthropologiques constants de la rationalité occidentale. Mais pourquoi aller jusqu'à affirmer que le carno-phallogocentrisme constitue 1' «armature cachée » de celle-ci?

Dans Par-delà bien et mal (cf. $\S 17$ et $\S 20)$, Nietzsche attribuait ce travers dualiste et ses dérivés (métaphysique substantielle, incapacité de faire droit à la différence et au devenir...) à la grammaire des langues indo-européennes. Derrida reprendra cette idée, en parlant d'un «contrat entre la grammaire du sujet ou du substantif, et l'ontologie de la substance » foncièrement dualiste et négatrice de la «différance » (Derrida, «Manger» 277). Les philosophes écoféministes tentent de remonter plus loin, en s'interrogeant sur les raisons d'être de cette grammaire dualiste elle-même; c'est ici que le concept de carnophallogocentrisme apporte un éclairage percutant. Il ouvre en effet un soupçon : et si ces principes de notre logique et de notre grammaire, loin d'être neutres, étaient symptomatiques d'un point de vue politiquement situé, «l'identité de maître » — cette «identité culturelle complexe forgée dans le contexte des dominations croisées de classe, de race, d'espèce et de genre » (Plumwood, Feminism 68)? Tout comme Derrida creusant et infléchissant l'intuition de Nietzsche, Plumwood établit un lien entre les règles de la logique occidentale et l'organisation inégalitaire des sociétés dans lesquelles elle a été formalisée, notamment concernant les rapports de sexes, mais aussi d'espèces : n'y aurait-il pas un cercle vicieux entre la séparation hiérarchisée des classes, des sexes et des espèces, qui caractérisait les sociétés indo-européennes antiques, et la grammaire dualiste qu'elles ont élaborée, projetant la même structure sur l'ensemble des catégories mentales et linguistiques? Car «les dualismes ne sont pas juste des systèmes abstraits d'idées flottant librement dans 1'air; ils sont étroitement associés à la domination et à l'accumulation, et sont leurs expressions et justifications culturelles majeures » (42). Le dualisme serait donc ancré dans le centrisme, car «un dualisme résulte toujours d'un certain déni de la dépendance à l'égard d'être altérisés et subordonnés » (41) : il «traduit le monde en des termes correspondant au point de vue et aux intérêts de la catégorie "supérieure", du centre (Hartsock 1990). Il fournit l'ancrage culturel nécessaire à l'hégémonie centriste de classe, comme l'ont montré Gramsci et d'autres, ainsi qu'à l'androcentrisme, à 
l'eurocentrisme et à l'anthropocentrisme» (55). Ainsi, même si «le caractère genré des dualismes demeure généralement à l'arrière-plan, sous une forme non examinée et occultée » (44), il n'en serait pas moins essentiel à leur constitution; idem pour leur caractère insidieusement anthropocentriste. Mettre au jour et étudier frontalement ces biais politiques centristes : tel est l'apport spécifique de l'écoféminisme à la déconstruction critique de la rationalité occidentale.

Plumwood argumente plus précisément cette hypothèse d'une relation entre la logique dualiste qui sous-tend l'ensemble de la rationalité occidentale et l'identité de maître carno-phallogocentrée, en étudiant le type de négation en vigueur dans les langues indo-européennes : « dans la logique classique, la négation (non- $p$ ) est interprétée comme l'univers moins $p$, absolument tout dans l'univers sauf ce que recouvre $p »$ (56). Comme elle le dénonce, notre logique est «incapable de faire des distinctions plus fines » (57) que $p$ vs non- $p$. De ce fait, non- $p$ est homogénéisé, marginalisé, exclu comme absolument autre (hyperséparation) et ramené à l'arrière-plan, disponible pour son instrumentalisation - conformément aux cinq schèmes du dualisme exposés ci-dessus. Or, ce modèle défectueux de négation correspondrait parfaitement au point de vue autocentré du «maître », qui prend la position centrale du «même » comme allant de soi et qui construit tout le reste sur un mode dualiste comme «l'autre », un ensemble indifférencié, incommensurablement opposé et inférieur. Le type de négation en vigueur dans notre logique s'ancrerait donc « dans la perspective du pouvoir [...], qui place un sujet omnipotent au centre et qui construit les autres, marginalisés, à partir d'un ensemble de propriétés négatives » (44). Cette construction dualiste de la négation, loin d'être accidentelle, aurait donc partie liée avec l'androcentrisme et le patriarcat, comme le souligne Plumwood qui, commentant la forme du diagramme de Venn, ironise sur le «drame phallique de cette conception centriste de non-p » (57). Elle serait également intimement corrélée avec l'anthropocentrisme et le spécisme, comme le montre Derrida avec son concept d' « animot » destiné à dénoncer l'homogénéisation de l'immense diversité animale, réduite par cette forme de négation à du simple «non humain» indifférencié et amalgamé.

Le concept de carno-phallogocentrisme permet donc de mettre le doigt sur les fondements et les enjeux inattendus de notre logique, et de poser en des termes originaux la nécessité de la dépasser. En ce sens, l'écoféminisme entend aller significativement plus loin que les autres formes de féminisme - qu'il s'agisse du féminisme libéral, socialiste ou culturel. Car ceux-ci, même s'ils critiquent parfois le contenu particulier et les effets des dualismes hommes / femmes, corps /esprit ou sujet / objet, ne critiquent pas la matrice logique elle-même qui les sous-tend. Ils en perçoivent bien les conséquences dévastatrices, mais ils ne vont pas 
jusqu'à attaquer frontalement le paradigme conceptuel même, persistant au contraire à opposer et à hiérarchiser les termes différents. Pour les féminismes libéral et socialiste, il s'agit simplement de déplacer la frontière, afin d'intégrer les femmes et autres groupes infériorisés à la catégorie de sujet humain à part entière, toujours identifié à la transcendance, à la raison et à la culture — sans, donc, que les concepts ni la forme logique de leur relation ne soient transformés; quant au féminisme culturel, il se contente d'une inversion axiologique (entre hommes et femmes, ou nature et culture), en maintenant lui aussi la structure formelle dualiste. Mais l'écoféminisme s'efforce de déconstruire le dualisme en tant que tel, cherchant une troisième voie délicate.

Il nous est impossible de développer dans l'espace de cet article les pistes requises pour élaborer cette nouvelle voie et pour dépasser la logique dualiste qui incruste malgré nous le carno-phallogocentrisme dans tous nos raisonnements, puisqu'il contamine jusqu'à nos langues, nos grammaires et nos catégories de pensée. Mais tel est bien le programme philosophique fondamental de l'écoféminisme: il ne cherche pas simplement à protéger «la nature » et «les animaux » ni à faire accéder «les femmes » à l'《autonomie », mais à déconstruire et à repenser complètement ces concepts mêmes, le réseau notionnel et la structure formelle qui les articule. Son projet profond - une «aventure dans une terra incognita » (Ruether, New Woman 159) - est donc impossible à formuler dans les termes et les habitudes de la langue ordinaire, imprégnée par la logique d'entendement qui reflète la «perspective du maître » (car «les perspectives de maître sont hégémoniques et façonnent les représentations culturelles » [Plumwood, Environmental Culture 99]).

C'est pourquoi, paradoxalement, les textes écoféministes les plus cohérents sont peut-être ceux qui inventent une nouvelle langue à la lisière de l'intelligible, qui créent des néologismes débridés et des syntaxes iconoclastes, brisant la digue traditionnellement érigée dans notre culture entre philosophie et poésie, argumentation et sensibilité, concept et mythe, savoir et magie. En effet, «comment effectuer le changement pour l'immanence? [...] Par la magie, [...] l'art de changer la conscience à volonté (Starhawk 39), «l'art de faire appel au pouvoir-du-dedans et de l'utiliser pour nous transformer, pour transformer notre communauté et notre culture » (17): «rien ne change en effet si la forme, la structure, le langage ne changent pas aussi. Pour travailler la magie, commençons par fabriquer de nouvelles métaphores. » (56) L'écoféminisme en appelle donc non seulement à bouleverser l'organisation sociopolitique des rapports entre les sexes, les espèces et les cultures, en favorisant des idéaux d'altermondialisme, de solidarité, de justice écologique, d'éthique du partenariat et de care, mais aussi à créer d'autres manières d'écrire et de penser, d'expérimenter, de désirer et de ressentir. Pour « décoloniser » les groupes sociaux opprimés, les peuples exploités, les femmes, les 
animaux et la nature, il est nécessaire de décoloniser notre propre pensée : tel est le sens de l'appel récurrent à la magie et à la spiritualité, pour le moins surprenant dans un contexte philosophique. Mais alors le dépassement du carno-phallogocentrisme ne serait-il pas un dépassement de la philosophie elle-même?

\section{Textes cités}

BATAILle, Georges, L'érotisme, Paris, Minuit, 1957.

BEAuvorr, Simone de, Le deuxième sexe, Paris, Gallimard, 1949.

BoOKCHIn, Murray, Une société à refaire. Vers une écologie de la liberte, trad. C. Barret, Montréal, Écosociété, 2011.

Callicott, John Baird, Pensées de la terre. La nature dans les cultures du monde, trad. P. Madelin, Paris, éd. Wild Project, 2011.

DALY, Mary, Notes pour une ontologie du féminisme radical, trad. M. Causse, Québec, L’Intégrale éditrice, 1982.

D’EAubonne, Françoise, Le féminisme ou la mort, Paris, Pierre Horay éd., 1974.

—, Écologie/Féminisme. Révolution ou mutation? Paris, A.T.P., 1978.

DERRIDA, Jacques, L'animal que donc je suis, Paris, Galilée, 2006.

—, «Il faut bien manger — ou le calcul du sujet. Entretien avec JeanLuc Nancy », in Derrida, Points de suspension. Entretiens, Paris, Galilée, 1992, p. 269-303.

—, Séminaire. La bête et le souverain, vol. 1 (2001-2002), Paris, Galilée, 2008.

Descola, Philippe, Par-delà nature et culture, Paris, Gallimard, 2005.

Douglas, Mary, De la souillure, trad. A. Guérin, Paris, La Découverte, 2001.

EATON, Heather et Lois Ann LORENTZEN (dir.), Ecofeminism and Globalization. Exploring Context, Culture, and Religion, Lanham, Rowman and Littlefield Publishers, 2003. 
FEDERICI, Silvia, Caliban et la sorcière. Femmes, corps et accumulation primitive, trad. Collectif Senonevero, Genève/Paris, Entremonde, 2014.

Fraiman, Susan, «Pussy Panic vs Linking Animal. Tracking Gender in Animal Studies », Critical Inquiry, n 39, 2012, p. 89-115.

GAARD, Greta, «Toward a Queer Ecofeminism », Hypatia, vol. 12, $\mathrm{n}^{\circ} 1$, 1997, p. 114-137.

—, «Ecofeminism Revisited. Rejecting Essentialism and Re-placing Species in a Material Feminist Environmentalism », Feminist Formations, vol. 23, n 2, 2011, p. 26-53.

HerITIER, Françoise, Masculin / Féminin, vol. 2, Paris, Odile Jacob, 2002.

MAthiEU, Nicole-Claude, «Homme-culture et femme-nature?», L'Homme, vol. 13, n 3, 1973, p. 101-113.

MIES, Maria, Patriarchy and Accumulation on a World Scale, Londres, Zed Books, 1986.

MIES, Maria et Vandana SHIVA, Écoféminisme, trad. É. Rubinstein, Paris, L'Harmattan, 1998.

Mies, Maria et Veronika Bernholdt-Thomsen, The Subsistence Perspective. Beyond Globalised Economy, Londres, Zed Books, 1999.

NiETzSCHE, Friedrich, Par-delà bien et mal, trad. P. Wotling, Paris, GF, 2000.

ORTNER, Sherry, "Is Female to Male as Nature is to Culture? », in M. ZimBALIST RosALDO et L. LAMPHERE (dir.), Woman, Culture, and Society, Stanford, Stanford University Press, 1974, p. 68-87.

PARMENIDE, Fragments, in J. VOILQUIN (dir.), Les penseurs grecs avant Socrate, Paris, GF, 1964.

Plumwood, Val, Feminism and the Mastery of Nature, Londres, Routledge, 1993.

-, Environmental Culture. The Ecological Crisis of Reason, Londres, Routledge, 2001. 
RuETHER, Rosemary Radford, Gaia and God. An Ecofeminist Theology of Earth Healing, New York, Harper \& Collins, 1992.

- New Woman, New Earth. Sexist Ideologies and Human Liberation, New York, Seabury Press, 1975.

SAlleH, Ariel, Ecofeminism as Politics. Nature, Marx, and the Postmodern, Londres, Zed Books, 1997.

StARHAWK, Femmes, magie et politique, trad. Morbic, Paris, Les empêcheurs de penser en rond, 2003.

WARrEN, Karen, «The Power and the Promise of Ecological Feminism », Environmental Ethics, vol. 12, n 3, 1990, p. 126-145. 